\title{
Oxycodone-Mediated Activation of the Mu Opioid Receptor Reduces Whole Brain Functional Connectivity in Mice
}

\author{
Md Taufiq Nasseef, ${ }^{\dagger}$ Jai Puneet Singh, ${ }^{\dagger}$ Aliza T. Ehrlich, ${ }^{\dagger}$ Michael McNicholas, ${ }^{\dagger}$ Da Woon Park, ${ }^{\dagger}$ \\ Weiya $\mathrm{Ma}{ }^{\dagger}$ Praveen Kulkarni, ${ }^{\dagger}$ Brigitte L. Kieffer, ${ }^{*}{ }^{\dagger}$ and Emmanuel Darcq ${ }^{*}, \dagger$ \\ ${ }^{\dagger}$ Douglas Hospital Research Center, Department of Psychiatry, School of Medicine, McGill University, Montreal, Quebec H4H 1R3, \\ Canada \\ *Center for Translational Neuro-Imaging, Northeastern University, Boston, Massachusetts 02115, United States
}

Supporting Information

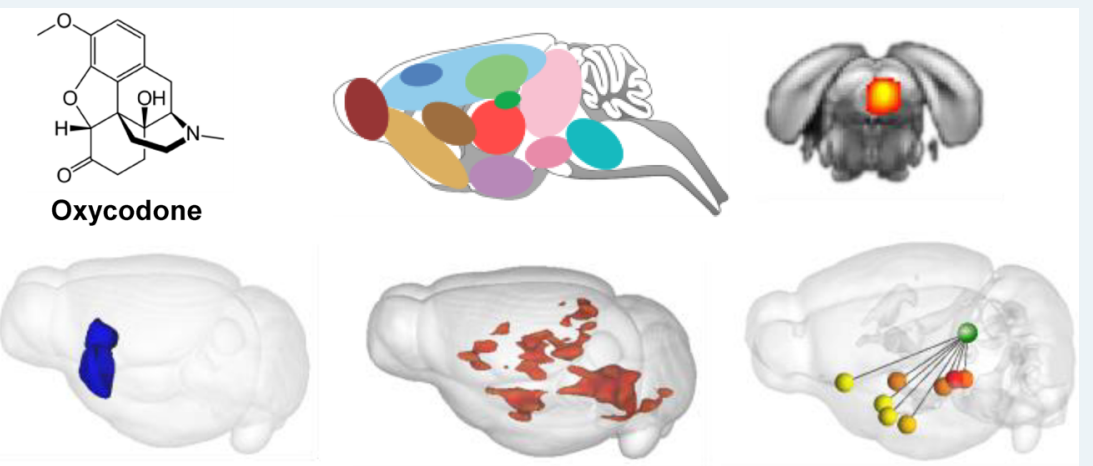

ABSTRACT: Oxycodone is a potent medicinal opioid analgesic to treat pain. It is also addictive and a main cause for the current opioid crisis. At present, the impact of oxycodone on coordinated brain network activities, and contribution of the mu opioid receptor (MOR) to these effects, is unknown. We used pharmacological magnetic resonance imaging in mice to characterize MOR-mediated oxycodone effects on whole-brain functional connectivity (FC). Control (CTL) and MOR knockout (KO) animals were imaged under dexmedetomidine in a 7Tesla scanner. Acquisition was performed continuously before and after $2 \mathrm{mg} / \mathrm{kg}$ oxycodone administration (analgesic in CTL mice). Independent component analysis (data-driven) produced a correlation matrix, showing widespread oxycodone-induced reduction of FC across 71 components. Isocortex, nucleus accumbens (NAc), pontine reticular nucleus, and periacqueducal gray (PAG) components showed the highest number of significant changes. Seed-to-voxel FC analysis (hypothesis-driven) was then focused on PAG and NAc considered key pain and reward centers. The two seeds showed reduced FC with 8 and 22 Allen Brain Atlas-based regions, respectively, in CTL but not KO mice. Further seed-to-seed quantification showed highest FC modifications of both PAG and NAc seeds with hypothalamic and amygdalar areas, as well as between them, revealing the strongest impact across reward and aversion/pain centers of the brain. In conclusion, we demonstrate that oxycodone reduces brain communication in a MOR-dependent manner, and establish a preliminary whole-brain FC signature of oxycodone. This proof-of-principle study provides a unique platform and reference data set to test other MOR opioid agonists and perhaps discover new mechanisms and FC biomarkers predicting safer analgesics.

KEYWORDS: oxycodone, mu opioid receptor, fMRI, BOLD activation, functional connectivity, ICA

\section{INTRODUCTION}

Oxycodone, a semisynthetic opioid that was first made from thebaine and introduced in clinical practice a century ago. This drug is a most potent opioid analgesic widely used to reduce acute or chronic pain, ${ }^{1}$ and oxycodone utility in the clinic has been extensively documented. ${ }^{2-5}$ In fact, oxycodone shows pain-relieving effects comparable to morphine, although with distinct pharmacological properties that include lower receptor binding, higher oral bioavailability, faster onset, and longer duration of action. ${ }^{6-8}$ As for morphine also, oxycodone is strongly addictive ${ }^{9,10}$ and is a controlled substance. In the past decade, the overprescription of opioid analgesics for pain treatment, including oxycodone, has led to an alarming increase of transition to heroin or fentanyl abuse and deaths by opioid overdose, the so-called opioid crisis. ${ }^{11,12}$ There is an urgent need to better understand opioid effects on the brain and develop safer opioid analgesics. ${ }^{13-15}$

Oxycodone, as well as other used and/or abused opioid drugs, produces analgesia, euphoria, and respiratory depression by activating the mu opioid receptor (MOR), considered the primary molecular target. ${ }^{16}$ This receptor is an inhibitory $G$

Received: April 1, 2019

Published: June 28, 2019 

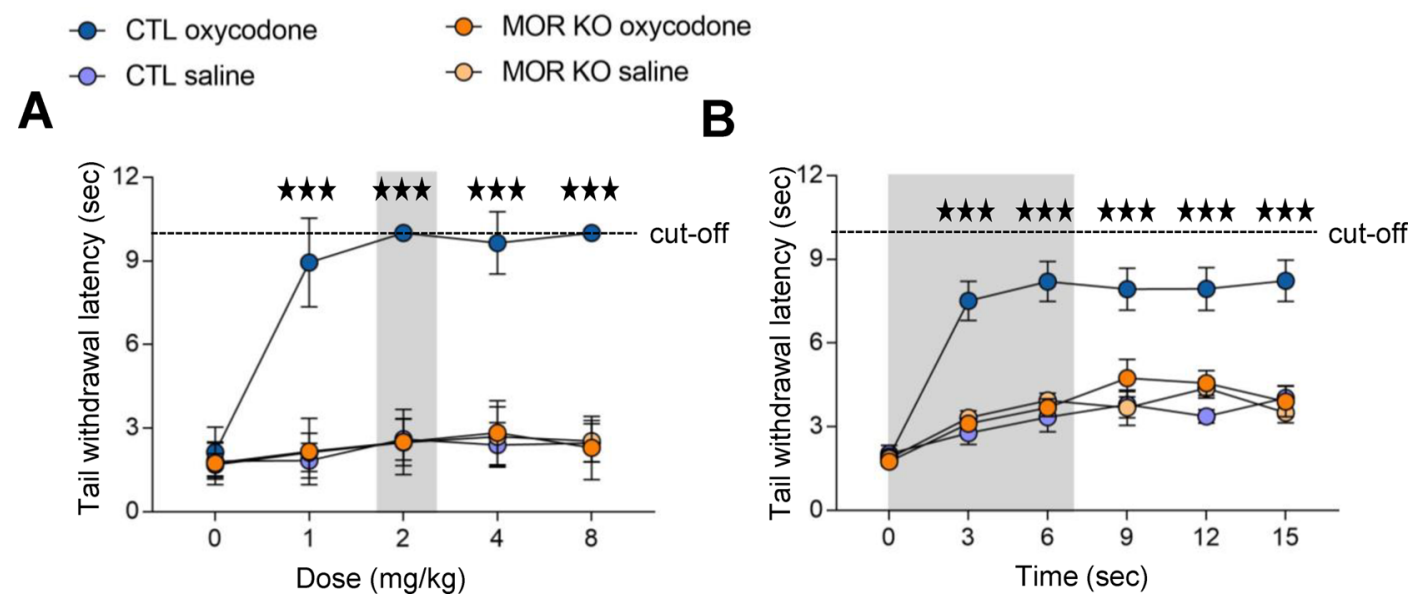

Figure 1. Analgesic effects of oxycodone. (A) Dose-response for analgesia. Analgesic effect of oxycodone was assessed with the tail immersion test. Tail withdrawal latency was measured at $52{ }^{\circ} \mathrm{C}, 15 \mathrm{~min}$ after oxycodone administration at 0 (saline), 1, 2, 4, and $8 \mathrm{mg} / \mathrm{kg}$ in MOR KO mice and their controls (CTLs) using a cumulative dose design. (B) Kinetics of analgesia. Tail withdrawal was measured every 3 min after injection of oxycodone $(2 \mathrm{mg} / \mathrm{kg})$ or saline. CTL $(n=10)$ and MOR KO $(n=10)$; RM-ANOVA with posthoc Newman-Keuls test: effect of the drug, $* * * p<$ 0.001. Light gray shadow covers the time period further used for the MRI acquisition and shows strong behavioral responses at the time of image analysis.

protein coupled receptor, ${ }^{17,18}$ broadly distributed throughout pain and reward circuits ${ }^{19,20}$ where it mediates both therapeutic and adverse effects of opioid drugs. ${ }^{13,16}$ In preclinical research, oxycodone activity on cellular signaling and behavior has been characterized in several animal models of pain, and some activity at other opioid receptors was also suggested (reviewed in ref 7). At present, however, the overall impact of oxycodone exposure on brain network activities and the specific MOR-mediated effects contributing to these effects remain poorly understood.

Magnetic resonance imaging (MRI)-based neuroimaging is a most informative noninvasive approach to study brain function and disease in humans (for opioid use disorders see review in ref 15 ). There is increasing interest in back-translating this approach to rodent research in order to scale-up drug testing, provide mechanistic insights into drug effects on whole-brain activity, and ultimately enhance the success rate of drug discovery in CNS research. ${ }^{21}$ Pharmacological MRI has now been developed in rodents, ${ }^{22}$ and although at an early stage, should be highly powerful to understand brain responses to drugs in healthy animals or disease models. ${ }^{23,24}$

A recent study mapped oxycodone-induced blood oxygen level dependent (BOLD) modifications in the rat, ${ }^{25}$ and our own previous study in the mouse compared oxycodone effects in mice lacking the Oprm1 gene (encoding MOR) and their controls, to reveal MOR-mediated drug effects on the BOLD signal. ${ }^{26}$ Still, the influence of oxycodone on coordinated brain network activities-also called functional connectivity (FC) has not been investigated in rodents. In the present study, we developed a new experimental procedure to characterize FC alterations upon acute oxycodone exposure, which are specifically mediated by MOR. Our analysis using both open-ended on the whole brain and seed-based analytic approaches focused on pain and reward-related seeds provides an oxycodone signature on whole-brain functional connectivity (WBFC), and reveals major modifications in key pain and reward centers.

\section{RESULTS}

Oxycodone Dose for fMRI. To select a dose for the FC study, we investigated behavioral effects of oxycodone in our mouse strain. We first tested oxycodone analgesia in mice lacking the Oprm1 gene (MOR KO) and their controls (CTL) using the tail immersion test (Figure 1A). Analgesic effects were measured at four doses of oxycodone using a cumulative dosing design ( $0,1,2,4,8 \mathrm{mg} / \mathrm{kg}$, see Methods), where oxycodone was administered, then tail withdrawal latency was measured after $15 \mathrm{~min}$, after which the next dose was administered. Saline injections were used as controls. Three way RM-ANOVA revealed significant main effects of both oxycodone doses $\left(F_{(4 ; 144)}=7.99 ; p<0.001\right)$, genotype $\left(F_{(1 ; 36)}\right.$ $=146.92 ; p<0.001)$, and drug $\left(F_{(1 ; 36)}=184.57 ; p<0.001\right)$, as well as a significant interaction doses $\times$ drug $\times$ genotype $\left(F_{(4 ; 144)}=3.09 ; p<0.05\right)$. At $1,2,4$, and $8 \mathrm{mg} / \mathrm{kg}$ oxycodone doses, a significant increase of latencies was observed in CTL (SNK posthoc; all $p<0.001$ ) but not in MOR KO animals, indicating that the analgesic effect of oxycodone is mediated by the MOR under our experimental conditions. The $2 \mathrm{mg} / \mathrm{kg}$ dose was the lowest dose producing maximal analgesia and was selected for the imaging study. We then investigated the kinetics of oxycodone analgesia at this dose, using repeated measures of tail withdrawal latencies every $3 \mathrm{~min}$ during 15 min in CTL and MOR KO animals (Figure 1B). Three way RM-ANOVA revealed significant main effects of both time $\left(F_{(5 ; 180)}=3.09 ; p<0.001\right)$, genotype $\left(F_{(1 ; 36)}=20.31 ; p<\right.$ $0.001)$, and drug $\left(F_{(1 ; 36)}=32.26 ; p<0.001\right)$, as well as a significant interaction time $\times$ drug $\times$ genotype $\left(F_{(5 ; 180)}=6.45\right.$; $p<0.001)$. Posthoc analysis indicated that oxycodone analgesia in CTL animals was rapid and reached significance at the 3 min time point (SNK posthoc: $p<0.001$ ). Again, and as expected, no analgesia was observed in MOR KO mice.

Altogether, based on the dose-response and kinetics of oxycodone analgesic, we chose to acquire MRI data during 7 min after oxycodone administration at the $2 \mathrm{mg} / \mathrm{kg}$ dose. This dosing and timing produces maximal analgesia in CTL mice.

Oxycodone Modifies the Blood Oxygen Level Dependent (BOLD) Signal. Animals were subjected to an optimized $^{26}$ scanning protocol $(\mathrm{TR}=1.65)$ in which 
A

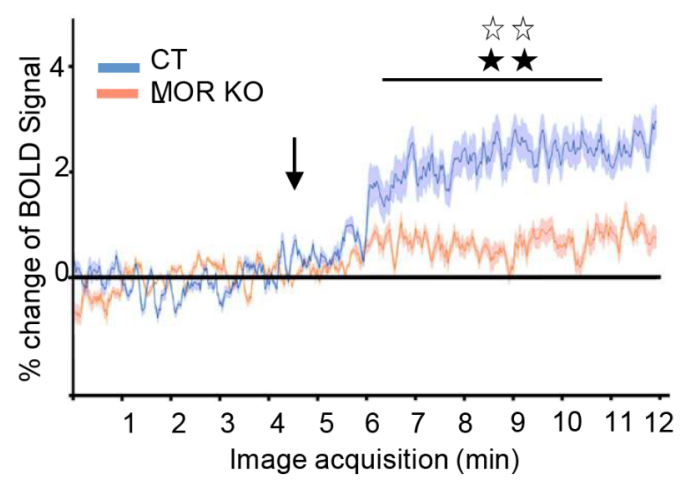

C
B

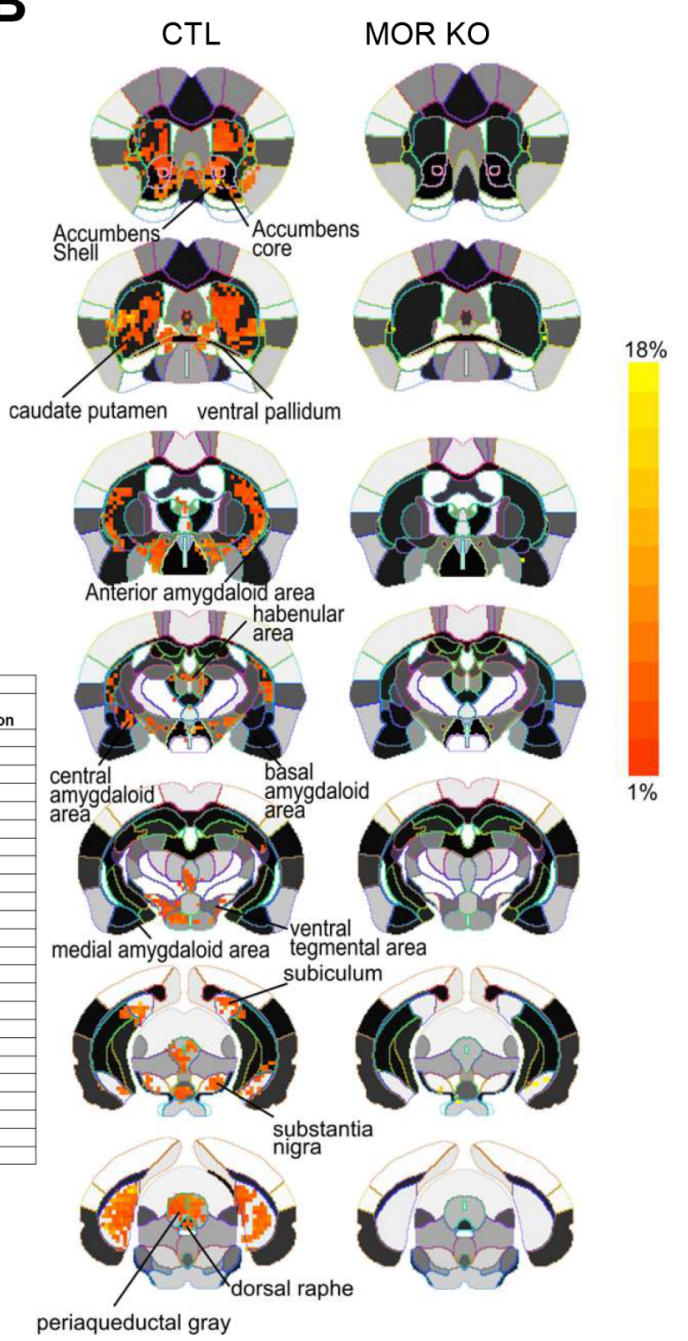

Figure 2. Modification of the BOLD signal upon oxycodone administration. (A) Oxycodone injection ( $2 \mathrm{mg} / \mathrm{kg}$ ) in the scanner (black arrow) increases the BOLD signal over time in the entire MOR cluster (Figure S2C-Sn) for CTL but not MOR KO mice. CTL $(n=10)$ and MOR KO ( $n$ $=10)$; Kruskal-Wallis nonparametric test, $* * p<0.01$. (B) BOLD activation maps for the MOR cluster $(2$ to 7 min after oxycodone administration) show increased signal in CTL (left) but not MOR KO (right) mice. Voxels showing an increase of BOLD after oxycodone in CTL mice were found at the level of nucleus accumbens core and shell, in caudate putamen, in hypothalamus, in amygdala, in interpeducular nucleus, dorsal raphe, and periaqueductal gray. No significant change was detected in MOR KO animals. (C) BOLD differences induced by oxycodone in each of the 23 regions of the MOR cluster. Table showing the total number of voxels composing the seed (Size), the average number of significant voxels \pm SEM for each region of the MOR cluster and the percent voxels with significant changes in BOLD activation after oxycodone administration in this region (\%) for CTL and MOR KO mice. Genotype effects for each region were analyzed using a Kruskal-Wallis test and regions were ranked in order of their significance.

acquisition was performed continuously 5 min before (baseline) and $7 \mathrm{~min}$ after oxycodone injection in the scanner (Supporting Information, Figure S1A-Sn and details in Methods). Preprocessing was done using a standard pipeline (semiautomated brain cropping, coregistration, motion correction, manual registration, smoothing, detrend, see Figure S1B-Sn and Methods) and postprocessing was done using both seed-based and voxelwise BOLD activation as described previously. ${ }^{26}$ We examined oxycodone-induced BOLD activation in four seeds (Figure S2A-Sn), including the periaqueductal gray (PAG) known as a major pain center of the brain, ${ }^{27,28}$ and three nuclei forming the mesolimbic dopaminergic pathway, namely the ventral tegmental area (VTA), the nucleus accumbens core (NAcc), and the nucleus accumbens shell (NAcs) considered essential for rewarding effects of drugs of abuse ${ }^{29,30}$ and in particular for opioids as they produce an increase of dopamine in the NAc via a disinhibition of the VTA dopaminergic neurons. ${ }^{31,32}$ As these brain regions are enriched in $\mathrm{MOR}^{19}$ and oxycodone is known to produce analgesic and rewarding effects, ${ }^{1,7,9}$ we hypothesized that oxycodone would alter the BOLD signal in the pain and reward centers. We found that oxycodone increased the positive BOLD signal in all these seed regions in CTL mice, and this was not observed in MOR KO animals (effect of oxycodone with the nonparametric Kruskall-Wallis test: in WT $=$ PAG $p<0.01$, VTA $p<0.05$, NAcc $p<0.01$, NAcs $p<0.01$ and in KO PAG $p=0.13$, VTA $p=0.52$, NAcc $p=0.13$, NAcs $p=0.17$ ) (Figure S2B-Sn). Further, there was a significant genotype difference, in the 2-7 min period following oxycodone administration, and for the four seeds (effect of genotype with the non-parametric Kruskall-Wallis test, PAG $p<0.01$, VTA $p=0.052$, NAcc $<0.01$, NAcs $p<0.01$ ). Data from 
A

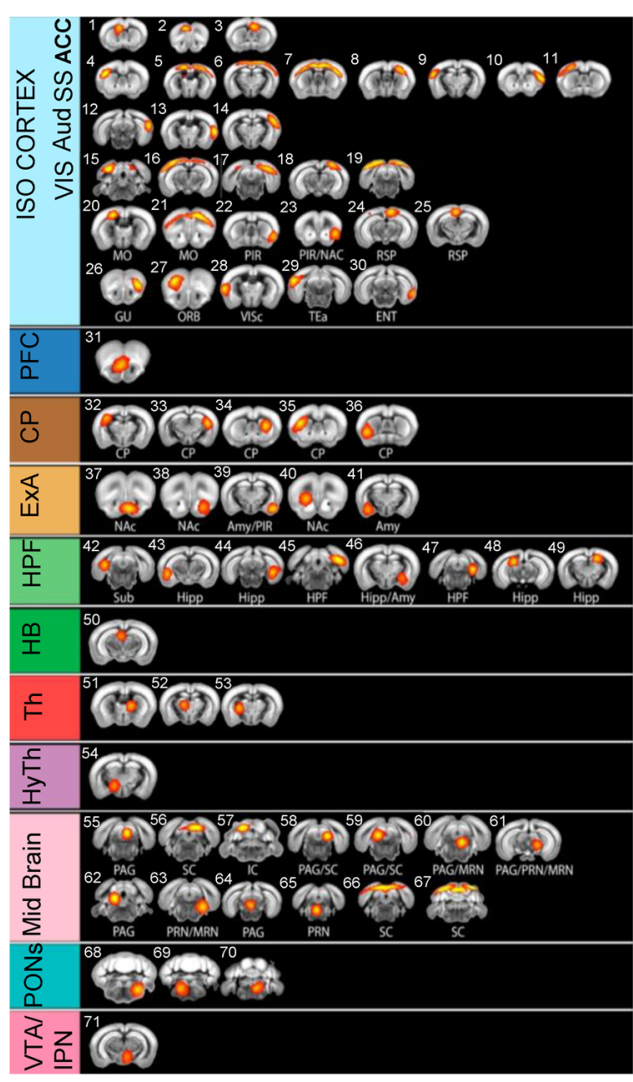

B

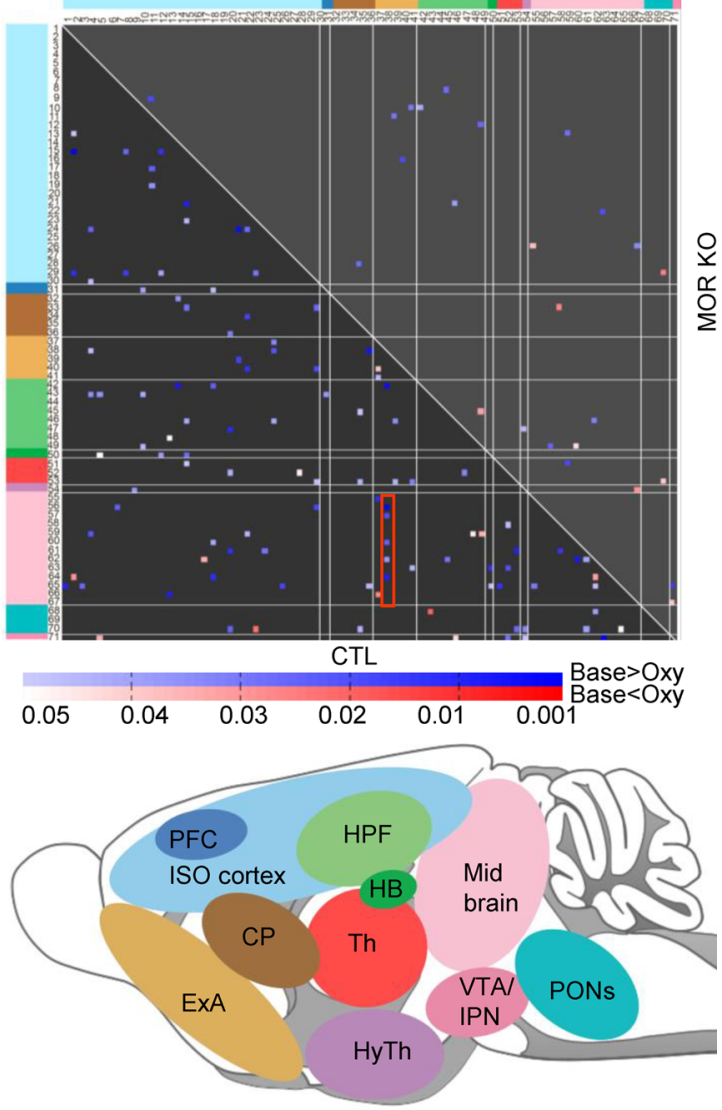

Figure 3. Independent component analysis of oxycodone effects on whole brain functional connectivity. (A) Spatial ICA performed on the pooled data sets (CTL baseline, CTL oxycodone, MOR KO baseline, MOR KO oxycodone) identifies 71 anatomically well-defined components, which are displayed in the rostrocaudal order, based on their correspondence with larger brain areas shown on the sagittal scheme (bottom right, 11 colorcoded brain areas); (B) Oxycodone effects on WBFC. The correlation matrix represents significant FC differences induced by oxycodone administration across the 71 components in CTL (lower triangle) and in MOR KO (upper triangle) groups. Blue and red scaling color bars indicate decreased or increased oxycodone-induced FC modification, respectively. The red rectangle is pointing to component 38, which overlaps with NAc and shows FC changes with five midbrain components. This is one example of top components with the highest number of FC modifications. CTL $(n=10)$, MOR KO $(n=10)$. CP = caudate putamen; ExA = extended amygdala; HB = habenula; HPF = hippocampal formation; $\mathrm{HyTh}=$ hypothalamus; $\mathrm{Th}=$ thalamus.

individual seeds, therefore, demonstrate that positive BOLD increases throughout brain regions mediating pain and/or reward, and that the modification is mediated by MOR.

To further test whether oxycodone more generally modifies the BOLD signal in MOR-enriched regions, we created a MOR cluster by pooling 23 MOR-enriched seeds (see Methods and $3 \mathrm{D}$ representation in Figure S2C-Sn) and quantified the overall BOLD signal in this cluster along image acquisition. As for the four individual brain regions, the positive BOLD signal increased in CTL mice as soon as $2 \mathrm{~min}$ after oxycodone administration, and this was not observed for MOR KO mice (Figure 2A; effect of oxycodone with thenon-parametric Kruskall-Wallis test: in WT $p<0.01$ and in KO $p=0.49$ ). These observations indicate that oxycodone modifies the BOLD signal, and that this effect is MOR-mediated under our experimental conditions. Then, we mapped the voxels showing a significant increase upon the oxycodone challenge in CTL (Figure 2B). No modification was detected in MOR KO mice (Figure 2B). For each of the 23 regions of the MOR cluster, we counted the number of voxels including mean showing a significant increase of BOLD after oxycodone administration (Figure 2C). We ranked the regions by significance levels for genotype effect (nonparametric Kruskall-Wallis) and found that BOLD activity is modified by oxycodone in 18 regions out of $23(78 \%)$. Thus, we demonstrate that oxycodone broadly alters BOLD activity in a MOR-dependent manner.

Data-Driven Independent Component Analysis (ICA) Reveals Oxycodone Effects on Whole Brain Functional Connectivity (WBFC). For further FC analysis, we considered four data sets: CTL $(n=10)$ and MOR KO $(n=10)$ groups at baseline (0-5 $\mathrm{min})$ and after oxycodone administration (2-7 min postinjection). We applied data-driven spatial independent component analysis (100-ICASSO) combining all the four subgroups (e.g., CTL baseline, CTL oxycodone, KO baseline, and $\mathrm{KO}$ oxycodone; see Methods section) and generated 100 components. We validated the quality, stability, and reliability of these 100 components using the ICASSO toolkit. Forty percent of the components showed a high quality index ${ }^{33} 0.9$, indicating high stability of these components (Figure S3-Sn). All the components were identified and annotated using the Allen Mouse Brain Atlas (2011) and ranked by affiliation with larger brain areas. Components with low quality or belonging to the olfactory bulb and cerebellum were removed from the analysis, and we finally used 71 components for further analysis (Figure 3A). 


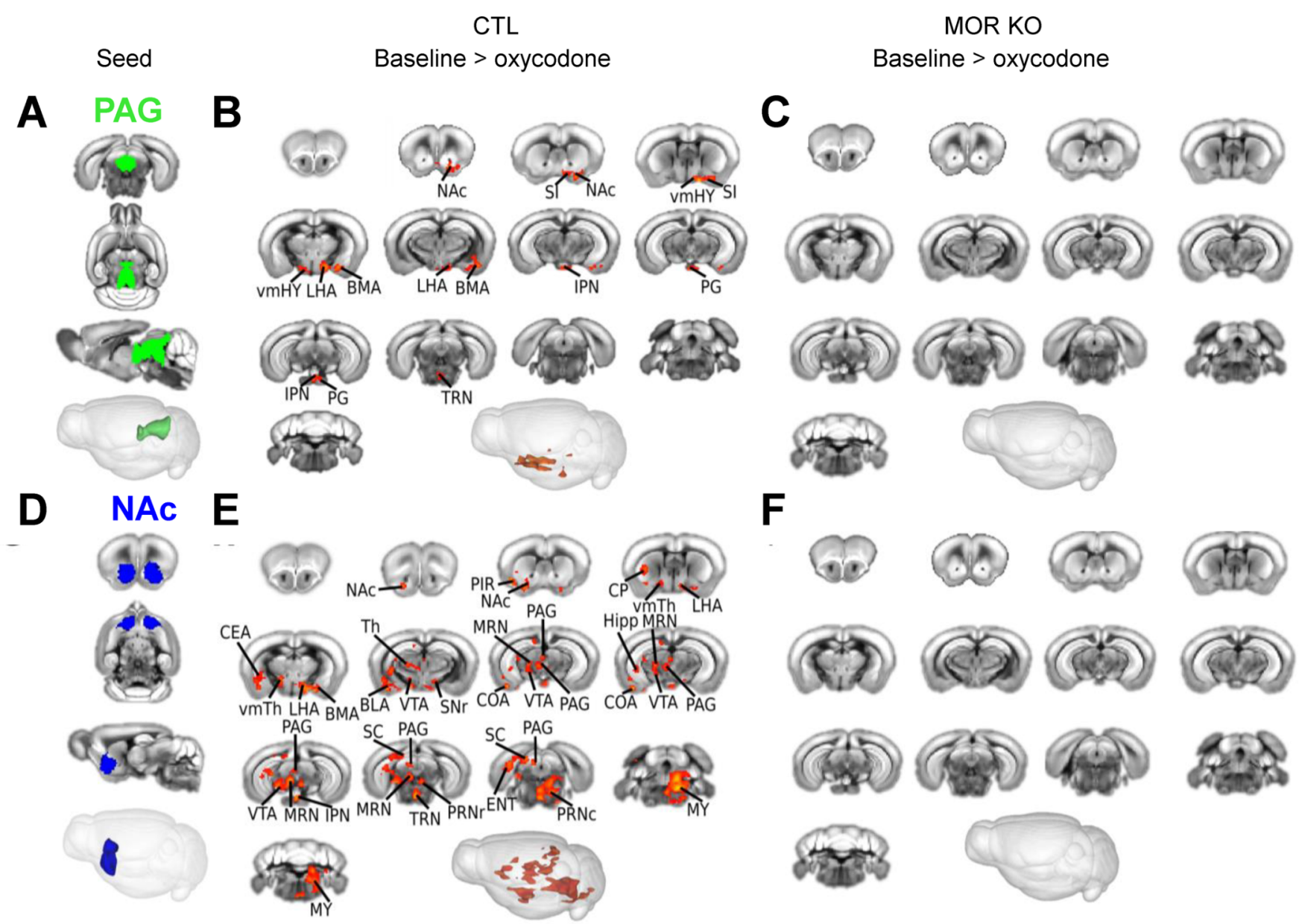

$\mathrm{T}$

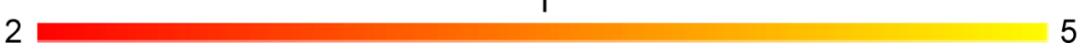

Figure 4. Seed-based voxelwise analysis of oxycodone effects on PAG and NAc functional connectivity. (A-C) Periaqueductal gray (PAG) seedbased analysis to identify oxycodone-induced FC alterations of PAG with the rest of the brain. (A) 2D and 3D representation of the PAG seed created from the Allen Mouse Brain Atlas (2011). (B) PAG connectivity is significantly reduced with eight annotated brain regions in CTL but not in MOR KO (C) mice. (D-F) Nucleus accumbens (NAc) seed-based analysis to identify oxycodone-induced FC alterations of NAc with the rest of the brain. (D) 2D and 3D representation of the NAc seed created from the Allen Mouse Brain Atlas (2011). (E) NAc connectivity is significantly reduced with 22 annotated brain regions in CTL but not in MOR KO (F) mice. (B-E) Red to yellow voxels represent significant decrease of FC (group comparison (baseline versus oxycodone) by $t$ test $(T$ values $>2, \mathrm{cc}=0.05$, CTL $(n=10)$, MOR KO $(n=10)$, FWER corrected). [BLA $=$ basolateral amygdalar nucleus, $\mathrm{BMA}=$ basomedial amygdalar nucleus, $\mathrm{CP}=$ caudoputamen, $\mathrm{CEA}=$ central amygdalar nucleus, $\mathrm{COA}=$ cortical amygdalar area, ENT = entorhinal area, Hipp = hippocampus, IPN = interpeduncular nucleus, LHA = lateral hypothalamic area, $\mathrm{MRN}=$ midbrain reticular nucleus, $\mathrm{MY}=$ medulla, $\mathrm{PG}=$ pontine gray, $\mathrm{PIR}=$ piriform area, $\mathrm{POR}=$ superior olivary complex, $\mathrm{PRN}=$ pontine reticular nucleus/caudal part, $\mathrm{SC}=$ superior colliculus, $\mathrm{SI}=$ substantia innominata, $\mathrm{SNr}=$ substantia nigra, $\mathrm{TRN}=$ tegmental reticular nucleus, vmHy $=$ ventromedial hypothalamic nucleus, $\mathrm{vmTh}=$ ventral medial nucleus of the thalamus, $\mathrm{Th}=$ thalamus.]

FC between each component was analyzed for the four groups, that is, at baseline or after oxycodone administration, and in CTL or MOR KO animals, and group comparisons were performed. Figure $3 \mathrm{~B}$ shows the oxycodone effect in CTL (left triangle) and MOR KO (right triangle) mice. Data are shown as a matrix, with color-coded $p$ values for all the significant oxycodone-induced FC changes (one tail $t$ test $(p<$ 0.05). In CTL mice, we found frequent and widespread reduction of $\mathrm{FC}$ across the components (blue squares), while events of increased FC (red squares) were more limited. A total of 118 significant FC reductions (with $p$ values from 0.049 to 0.00029 ) and 17 significant FC augmentations (with $p$ values from 0.0093 to 0.00023 ) were detected, forming altogether an oxycodone fingerprint on WBFC. In MOR KO mice, oxycodone had little effect on WBFC (Figure 3B) and none of the changes in CTL mice was found in MOR KO mice, indicating that the oxycodone fingerprint is MORmediated.

Notable for example, was the high number of changes between component 43 (one of the 3 NAc components) and several midbrain components (five in total, highlighted in red in the matrix, Figure 3B). To quantify oxycodone effects on FC and highlight the most significant modifications, we further ranked the components by number of FC alterations (Table S1-Sn). The top four included components $15,38,65$, and 62, overlapping with visual cortex/inferior colliculus, NAc, pontine reticular nucleus, and PAG, respectively. Altogether, the datadriven ICA analysis demonstrates that oxycodone largely 


\section{A PAG Overlapping voxels}

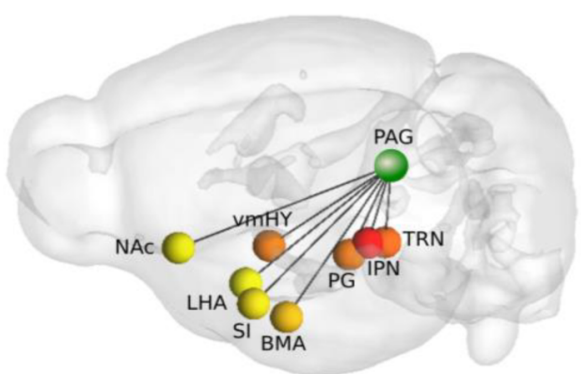

\section{B NAc}

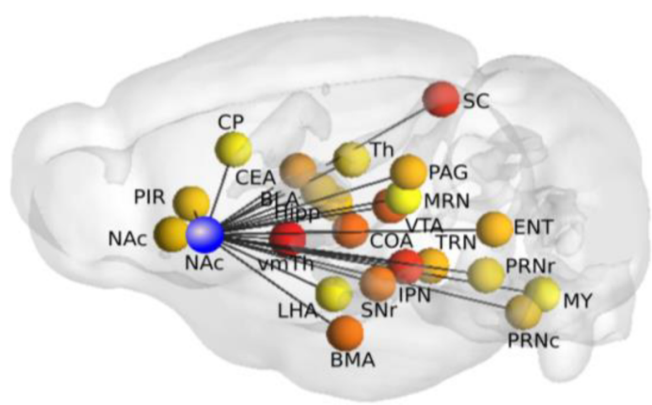

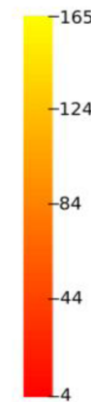
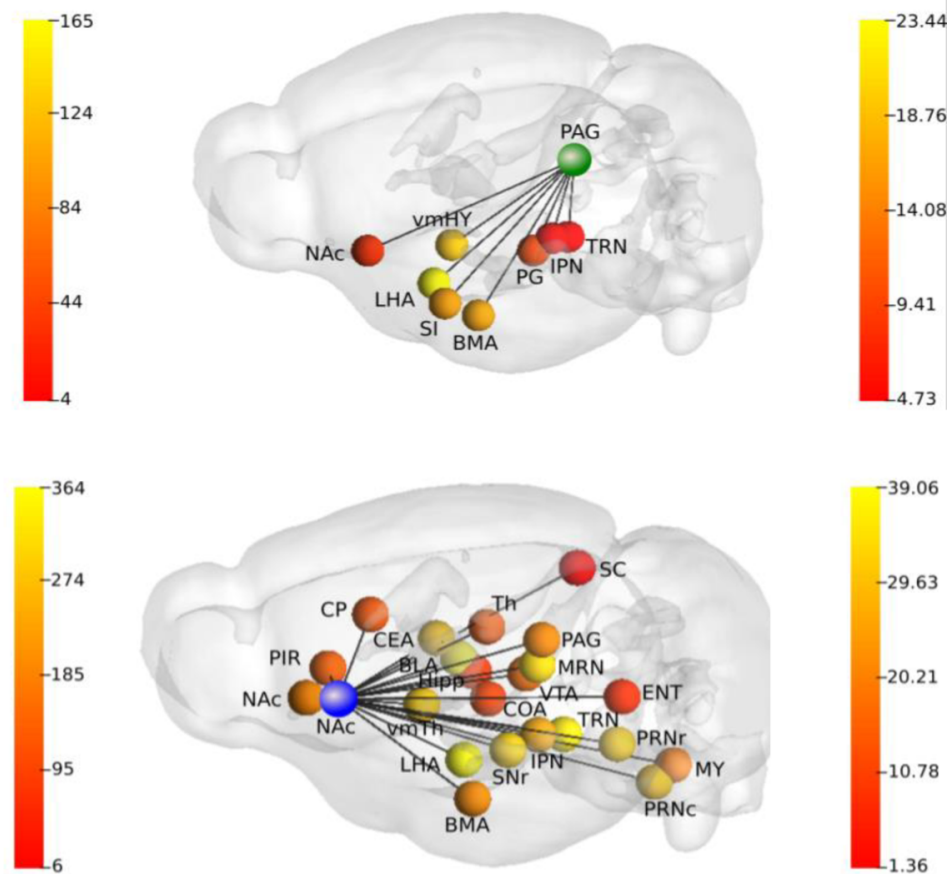

Percent voxels

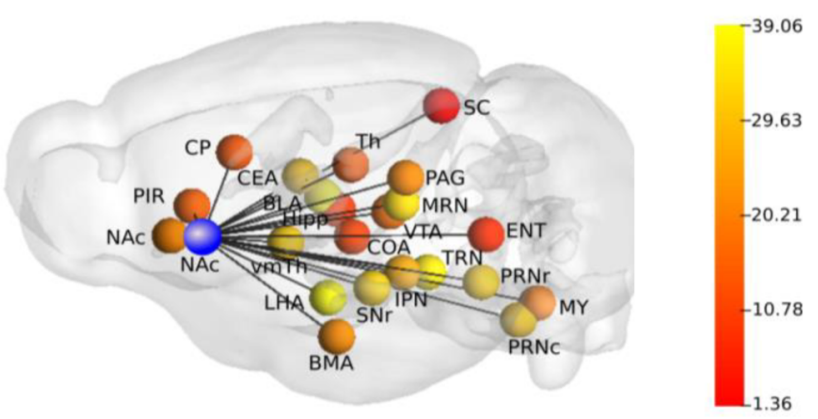

Figure 5. Quantification of oxycodone effects on functional connectivity of PAG and NAc seeds. 3D representation of PAG (A) and NAc (B) seeds, and their respective brainwide FC modifications. Scaled color bars show significance of the oxycodone effect expressed as number of overlapping voxels (left) or precent voxels (right) modified by oxycodone in the target seed (see also Tables S2-Sn and S3-Sn). Each target is represented by a sphere color-coded to show the level of FC reduction. CTL $(n=10)$, MOR KO $(n=10)$. [BLA = basolateral amygdalar nucleus, $\mathrm{BMA}=$ basomedial amygdalar nucleus, $\mathrm{CP}=$ caudoputamen, $\mathrm{CEA}=$ central amygdalar nucleus, $\mathrm{COA}=$ cortical amygdalar area, $\mathrm{ENT}=$ entorhinal area, Hipp = hippocampus, IPN = interpeduncular nucleus, $\mathrm{LHA}=$ lateral hypothalamic area, $\mathrm{MRN}=$ midbrain reticular nucleus, $\mathrm{MY}=$ medulla, $\mathrm{PG}=$ pontine gray, $\mathrm{PIR}=$ piriform area, $\mathrm{POR}=$ superior olivary complex, $\mathrm{PRN}=$ pontine reticular nucleus/caudal part, $\mathrm{SC}=$ superior colliculus, $\mathrm{SI}$ = substantia innominata, $\mathrm{SNr}=$ substantia nigra, $\mathrm{TRN}=$ tegmental reticular nucleus, $\mathrm{vmHy}=$ ventromedial hypothalamic nucleus, $\mathrm{vmTh}=$ ventral medial nucleus of the thalamus, $\mathrm{Th}=$ thalamus.]

modifies FC throughout the brain in a MOR-dependent manner, and induces the strongest FC modifications for components belonging to the cortex, as well as key reward (NAc) and pain (PAG) centers.

Seed-Based Voxelwise Analysis Reveals Reduced PAG and NAc FC with Several Midbrain Regions. Results from ICA data quantification prompted us to refine the analysis of major oxycodone-induced FC alterations using a hypothesis-driven approach. We thus selected NAc and PAG, two brain areas that show a strong BOLD signal (Figure 2C and Figure S2-Sn) and highest FC (Figure 3B and Table S1$\mathrm{Sn})$ modifications under oxycodone exposure, and also known to drive analgesic and addictive opioid effects. We manually generated a PAG and a NAc seed using the Allen Mouse Brain Atlas (2011) (Figure 4A,D) and examined whole brain voxelwise FC for these seeds. In CTL groups, we found that oxycodone significantly $(p<0.05$, FWER correction) reduces FC for the two seeds, and more modifications were observed for the NAc seed compared to PAG (Figure 4B,E). PAG FC was reduced with at least 8 identifiable regions. These include the NAc, substantia innominate, amygdalar nuclei, interpeduncular nucleus, VTA, lateral hypothalamic area (LHA), and ventromedial hypothalamic nucleus (Figure 4B). For the NAc seed, we identified 22 regions showing reduced FC, including the NAc itself, caudate putamen, several amygdalar nuclei, entorhinal area, hippocampus, interpeduncular nucleus, LHA, midbrain reticular nucleus, pontine reticular nucleus/caudal part, superior colliculus, substantia nigra, PAG, tegmental reticular nucleus, and ventral medial nucleus of the thalamus (Figure 4E). In the MOR KO group, there was no significant modification of brainwide FC for any of the seeds (Figure $4 \mathrm{C}, \mathrm{F})$. This observation is concordant with both BOLD and ICA analyses, and indicates that FC reduction in the CTL group is mediated by MOR, at least under our experimental conditions.

Next, we quantified the extent of FC reduction for PAG and NAc seeds in CTL animals. To do so, we created seeds for all the identified brain regions showing altered FC with PAG or NAc (Figure S4-Sn) using the method that we previously described. $^{34}$ For each target seed region, we counted the number of voxels showing significant FC difference with the corresponding PAG (Table S2-Sn) or NAc (Table S3-Sn) seed. We ranked target areas based the number or percent of voxels significantly modified by oxycodone (Tables S2-Sn and S3-Sn) and results are shown in Figure 5, which displays a 3Dbrain representation of the data. FC reduction of PAG was strongest with LHA, vmHY, BMA, SI, and NAc (Figure 5A). NAc FC was reduced with many brain regions, but strongest modifications were with LHA and BLA (Figure 5B). Note that, in the NAc seed analysis, FC was also significantly reduced with PAG, indicating together that a decrease in communication between PAG and NAc is consistently observed independently from the seed considered. 
Altogether the seed analysis concurs with the ICA analysis to show that oxycodone reduces brain communication, and identifies a set of specific FC alterations for PAG and NAc seeds with ventral and posterior brain areas, respectively, as well as between the two seeds. Again, these effects of oxycodone are MOR-dependent.

\section{DISCUSSION}

In summary, both unbiased (ICA) and seed-based analytic approaches reveal that an analgesic dose of oxycodone triggers significant modifications of FC across the brain. Our data show that oxycodone produces essentially a reduction of communication between brain regions. This effect is widespread and, remarkably, primarily alters the function of PAG and NAc representing major pain ${ }^{27,28}$ and reward ${ }^{13,20}$ centers, respectively.

These changes occur in a time frame fully compatible with the kinetics of oxycodone effects at the behavioral level, as maximal modification of the BOLD signal is reached 2 min after oxycodone administration (Figure 2A), while maximal analgesic is achieved 3 to $6 \mathrm{~min}$ after injection, respectively (Figure 1). Altogether, therefore, our data characterize a preliminary oxycodone signature on WBFC in the mouse brain, which correlates temporally with behavioral effects of the drug. Further, a unique feature of this mouse study is the demonstration that the most oxycodone effects identified in this study are MOR-dependent. There was no detectable oxycodone effect in MOR KO mice for analgesic responses (Figure 1), BOLD activation (both temporal and spatial analyses, Figure 2) or seed-based FC (Figure 4). Scarce modifications were observed in the ICA-based analysis, the origin of which remains unclear and may relate to the very distinct postprocessing method sensitivity. We can reasonably assume that these FC differences do not reflect MORmediated oxycodone effects, as none of them was observed in CTL mice.

We first investigated oxycodone effects on the BOLD signal (brain activity), as classically done in earlier rodent studies. $^{25,26,35-37}$ One report showed that morphine $(5 \mathrm{mg} /$ $\mathrm{kg}$ ) increases BOLD activity in the hypothalamus, thalamus, amygdala, and PAG of anesthetized rats. ${ }^{37}$ Two other studies demonstrated that oxycodone $(2.5 \mathrm{mg} / \mathrm{kg})$ increases BOLD activity in the hypothalamus, thalamic areas, PAG, and the striatum in awake rats ${ }^{25}$ and mice, ${ }^{26}$ and altogether, increased BOLD activity was reported in several MOR-enriched brain areas important for pain and reward processes upon opioid exposure. $^{26,35-37}$ Here, we show that oxycodone $(2 \mathrm{mg} / \mathrm{kg}$ ) increases the BOLD signal in hypothalamic areas, PAG, and striatal areas (NAcc, NAcs, and CP), which is consistent with previous rodent findings. Note that two other studies found a morphine effect in similar brain areas (caudate nucleus in $\operatorname{dog} s^{39}$ and striatum in $\operatorname{rats}^{40}$ ). These authors reported a reduction of cerebral blood flow in these areas, contrasting our findings of increased BOLD signal perhaps due to the different nature of the opioid drug and imaging modalities. We further show increased BOLD in other areas including the subiculum, dorsal raphe, and $\mathrm{HB}$, suggesting that our methodology is highly sensitive. One-and the only-translational study comparing opioid-induced BOLD activity changes in rats and humans demonstrated that buprenorphine increases the BOLD signal in the subiculum, PAG, caudate putamen, and thalamic areas in both species. ${ }^{35}$ Remarkably, the subiculum, which is implicated in heroin seeking and addiction, ${ }^{38}$ is also the brain region showing strongest increased of BOLD activity in our study (Figure 2C). Altogether, our and previous data support the translatability potential of BOLD activity analyses for pharmacological MRI and the analysis of opioid effects on the brain.

To our knowledge, no animal study has examined the effects of opioids on communication within the brain. FC analytic methods were developed recently in rodents to study brain networks at rest or under stimulation, ${ }^{23,24}$ and represent a unique and innovative approach to study pharmacology in vivo, and at the level of the whole brain. ${ }^{24}$ Here, we investigated FC alterations upon an oxycodone challenge for the first time and give a proof-of-principle study. We first used an open-endedapproach to study oxycodone-induced FC modifications. ICA demonstrated that FC across functional nodes is essentially reduced by oxycodone, with the strongest effects for PAG and NAc components. This is a critical observation, which validates our approach, as alteration of PAG and NAc (ventral striatum) connectivity indeed parallels the major well-known behavioral oxycodone effects in mice (analgesia and reward). In humans, acute administration of oxycodone was also found to diminish FC, and disrupted anterior cingulate cortex (ACC)-insula and ACC-putamen (dorsal striatum) FC belonging to the pain matrix. ${ }^{41}$ To our knowledge, this study is the only report, which elucidates the effect of oxycodone on FC in healthy humans, and the analysis was particularly focused on pain networks. Our ICA analysis identified 3 ACC components (components 1-3) and their FC with isocortex (components 13,15 , and 29) and midbrain (component 65 ) was reduced by oxycodone (Figure $3 \mathrm{~B}$ ). In the future, it will be important to further develop brainwide analyses in both species, with more emphasis on cortical and subcortical networks, respectively, for mouse and human data sets in order to fully exploit the translatability potential of pharmacological MRI (PhMRI).

We further extended our open-ended ICA analysis with a seed-voxelwise based approach, and focused on the top-ranked areas relevant to pain (PAG) and reward (NAc). A first outcome of this aspect of the study is the spatial refinement in characterizing oxycodone-induced FC changes for the two seeds. As compared to the ICA component-based analysis, many more anatomically well-defined brain subregions are identified, which will further guide mechanistic circuit studies to understand oxycodone effects. Second, an interesting observation is that oxycodone does not only reduce FC of PAG and NAc seeds with other brain centers, but also reduces FC across the two seeds. This was detected using both the PAG- and the NAc-seed analysis (Figures 4 and 5) and is therefore a strong finding. At this stage, the significance of reduced communication between these two centers remains elusive, but pain and reward networks are intermingled, and pain relief is rewarding. We may speculate that a functional interaction between PAG and NAc, which is modified by oxycodone, contributes to circuits encoding the pleasurable effect of pain relief that are poorly characterized. ${ }^{42}$ Third, a remarkable observation is that oxycodone reduces FC of both PAG and NAc seeds with hypothalamic and amygdala regions, quantified as the top modified target seeds (Table S2-Sn, PAG to LHA, vmHY and BMA; Table S3-Sn, NAc to LHA and BMA). The data therefore reveal a strong impact of oxycodone in reducing communication across reward and aversion/pain centers of the brain. In the future, specific small network analyses involving these particular brain regions, and their correlation with behavioral scores, may be useful to better 
understand circuit mechanisms underlying biological activities of oxycodone, and also compare oxycodone effects with other opioid analgesics with lower abuse liability.

Finally, of note is the observation that FC is generally decreased upon oxycodone administration, while strengthened connectivity is barely observed in the ICA analysis and undetectable in seed-based analyses, a finding that may be linked to the well-established inhibitory action of MOR on neuronal activity. Testing other opioid drugs, and also the action of psychostimulant drugs under the same conditions should clarify this point in the future. In conclusion, our data provides a fully established methodology and a reference data set, which will facilitate the testing of other MOR opioids used in the clinic and under development. In the search for safer MOR analgesics, it will be important for example to determine whether MOR biased agonists, which preferentially induced G protein signaling over $\beta$-arrestin, and show less adverse effects (such as less euphoric response and respiratory depression), ${ }^{43}$ modify brain communication differently from traditional unbiased MOR agonists. Overall this study, focused on oxycodone, demonstrates the potential of PhMRI to establish opioid-induced FC patterns at the level of the entire brain, and perhaps discover new mechanisms and biomarkers that will allow the prediction of safer behavioral effects.

\section{METHODS}

Animals. Male CTL, MOR $\mathrm{KO}^{16}$ aged of 8-12 weeks were bred in-house at the Neurophenotyping Center of the McGill University/Douglas Hospital Research Institute and were produced as previously described. ${ }^{16}$ All experiments were performed following the guidelines and ethics on animal experimentation established by the Canadian Council of Animal Care and by the Animal Care Committees of McGill University/Douglas Mental Health University Institute, Montreal, Canada (protocol number 7466).

Behavioral Experiments. Oxycodone-Induced Analgesia. Mice were administered with oxycodone or saline and analgesia tested using the tail immersion test as previously described. ${ }^{16,44}$ Briefly, 15 min following oxycodone injections (for cumulative doses) or every $3 \mathrm{~min}$ during $15 \mathrm{~min}$ after oxycodone administration (for kinetics), the tail was immersed in a water bath set at $52{ }^{\circ} \mathrm{C}$ and tail withdrawal latencies were measured with a cutoff time of $10 \mathrm{~s}$. Mice received subcutaneous (SC) injections of cumulative doses of oxycodone $(0,1,2,4$, and $8 \mathrm{mg} / \mathrm{kg}$ ) every $15 \mathrm{~min}$ (for cumulative doses) or a single injections (SC) of $2 \mathrm{mg} / \mathrm{kg}$ of oxycodone (for kinetics).

For behavioral experiments, all statistical analyses were performed using Statistica (http://www.statsoft.com/ Products/STATISTICA-Features) with the repeated measure (RM) ANOVA and posthoc Newman-Keuls (SNK) test. Plotting was done using Prism (https://www.graphpad.com/ scientific-software/prism).

MRI Experiments. Preparation, Anesthesia, and Physiological Parameters. MRI data acquisition was achieved using a 7 Tesla small animal scanner (BioSpec 70/30USR, Bruker) using a RF SUC $3001 \mathrm{H}$ M.BR QSN RO AD (model number T12053 V3, Bruker) surface coil. Scanning was performed on 8 to 12 weeks old CTL and MOR KO male mice of 10 animals for each group under continuous dexmedetomidine (DMD, an $\alpha-2$ adrenergic agonist) sedation through a MRI compatible catheter (initial intraperitoneal injection of $0.15 \mathrm{mg}$ of DMD per kilogram of body weight in $100 \mu \mathrm{L}$ of $0.9 \% \mathrm{NaCl}$-solution followed by subcutaneous infusion of $0.3 \mathrm{mg}$ per kilogram of body weight in $200 \mu \mathrm{L} / \mathrm{h}$ ). The method was adapted from refs 45,46 . Isoflurane was used at $5 \%$ volume mixed with oxygen for 5 min to induce complete anesthesia in a chamber. Then isoflurane was reduced to $3 \%$ and dexmedetomidine bolus was injected i.p. for $2 \mathrm{~min}$, then animals were moved inside the scanner and dexmedetomidine only was administered SC. Body temperature during scanning was maintained using a warm air flow over the animal that was set at $37 \mathrm{C}$. Respiration rate was monitored and maintained between 60 and 90 breaths per minute using 1025-IBP-50 Small Animal Monitoring Gating System (SA instruments). After completing acquisition of the images, Antisedan (atipamezole hydrochloride, an $\alpha-2$ adrenergic antagonist) (Pfizer, Canada) was injected to improve animals' recovery. The dose of Antisedan was 0.05 $\mathrm{mg} / \mathrm{kg}$ diluted in Ringer's lactate solution.

Mouse Brain fMRI Data Acquisition. All fMRI images were obtained following Spin Echo EPI pulse sequence using a surface gradient coil with the following parameters: FOV, 4.28 $\times 1.32$; matrix, $128 \times 80$; slices, 20 ; resolution, $167 \times 165 \times$ $700 \mu \mathrm{m}^{3}$; TE/TR, $21 \mathrm{~ms} / 1.65 \mathrm{~s}$; flip angle, 70; repetitions, 450; total acquisition time, $12 \mathrm{~min}$ for each mouse. After an initial 5 min of resting state fMRI scan oxycodone was injected subcutaneously during images acquisition. Oxycodone was dissolved in $0.9 \% \mathrm{NaCl}$ and injected at a dose of $2 \mathrm{mg} / \mathrm{kg}^{26}$

MRI Data Analysis. BOLD Activation. For BOLD activation, all analyses were performed using Matlab SPM8 (http://www.fil.ion.ucl.ac.uk/spm/) and MIVA ${ }^{26}$ tool by following our previous studies. ${ }^{26,47}$ The images were cropped (identifying the nonbrain areas), coregistered with a mean functional image following SPM8 using the following parameters: quality, 0.97, smoothing, $0.3 \mathrm{~mm}$; separation, 0.5 $\mathrm{mm}$; and smoothed with a fwhm of $0.8 \mathrm{~mm}$ Gaussian kernel. Images were manually aligned and registered using the MIVA tool to a 3D mouse brain atlas, which was segmented and labeled with 140 discrete anatomical regions (Ekam Solutions, Boston MA) as described in our previous paper. ${ }^{26}$ The average value from all subjects within the group determined the composite value. With the use of voxel-based analysis, the percent change in BOLD signal for each independent voxel was averaged for all subjects. The first 187 repetitions $(5 \mathrm{~min}$ baseline) were considered as baseline and 188-450 repetitions (5-12 min) were considered as oxycodone effect. According to the distribution of MOR and its presence in structures involved in reward and pain processing, ${ }^{19}$ we selected $23 \mathrm{MOR}$ enriched regions and we called it the $\mathrm{Mu}$ Opioid cluster (containing the anterior olfactory, medial septal area, nucleus accumbens shell, nucleus accumbens core, bed nucleus stria terminalis, caudate putamen, central amygdaloid area, fornix, habenular area, lateral rostral hypothalamic area, paraventricular thalamic area, central medial thalamic area, lateral caudal hypothalamic area, dorsal medial hypothalamic area, medial mammillary area, substantia nigra, subiculum, interpeduncular area, periaqueductal gray, dorsal raphe, para brachial area, locus coeruleus, and ventral tegmental area; Figure 1D). Mean BOLD activation of the Mu Opioid cluster was presented to compare across two different groups, and statistical analysis was performed using $t$ test with FDR and cluster correction following our previous study. ${ }^{26}$ Then four brain regions of the $\mathrm{Mu}$ Opioid cluster (nucleus accumbens core, shell, ventral tegmental area and periaqueductal gray) were analyzed individually. For genotype effect, the nonparametric Kruskall-Wallis test were used to identify statistical 
differences between CTL and MOR KO after oxycodone administration. ${ }^{26}$ Moreover, the Kruskall-Wallis test was also applied to investigate the drug effect between the baseline and after oxycodone administration individually for CTL and MOR $\mathrm{KO}$.

fMRI Data Analysis. The functional connectivity preprocessing pipeline involved the following six steps: (i) denoising using Advanced Normalized tools (ANTs) (https://github. com/ANTsX/ANTs/blob/master/Examples/DenoiseImage. cxx); motion correction using ANTs and FSL (https://fsl. fmrib.ox.ac.uk/fsl/fslwiki); (iii) registration done by two steps, first normalizing all the subject's data using a template created by the existing subject's mean volumes using ANTs, and second registering all these normalized data with the highresolution Allen Mouse Brain Connectivity template $(2011)^{46}$ via MAGeT tool; ${ }^{48}$ (iv) smoothing with a Gaussian Kernel of full width half-maximum (fwhm) at 0.3 by AFNI (https://afni. nimh.nih.gov/); (v) cropping using a brain mask generated according to gray and white matter segmentation using Allen template space and applied to all subjects; (vi) bandpass filtering by AFNI tool at a frequency of window between 0.01 $\mathrm{Hz}$ to $0.1 \mathrm{~Hz}^{34}$

For postprocessing analysis, we applied two different methods: (i) independent component analysis (ICA) ${ }^{46}$ and seed-based analysis. ${ }^{31}$ First, ICA was performed using both CTL and MOR KO group subjects using GIFT tools (Group ICA of fMRI Toolbox, v4.0a, www.nitrc.org/projects/gift/). Moreover, to examine the stability and reliability of the population ICA patterns by randomization and bootstrapping in each experimental group for different number of components, ICASSO ${ }^{49}$ was applied. To quantify the measure of robustness of the components isolation and compactness, "Quality index ${ }^{33}$ (value range 0 to 1 )" was used for each cluster. ${ }^{46}$ Then, functional connectivity analysis was performed using FSL. Next, seed-based voxelwise analysis was performed to study whole-brain functional connectivity patterns of nucleus accumbens (NAc) and periaqueductal gray (PAG), as described in our previous work. ${ }^{34,50,51}$ In brief, each selected 3D bilateral seed was constructed manually following the Allen Mouse Brain Connectivity Atlas reference atlas (2011 Allen Institute for Brain Science. Allen Mouse Brain connectivity atlas API. Available from http://mouse.brain-map.org/ experiment/thumbnails/100048576?image_type=atlas). Correlation analysis was done as follows: (i) the mean of each seed time series was used to produce a whole brain correlation map, ${ }^{52}$ transformed into Fishers $\mathrm{Z}$ scores tested for significant connectivity by single $t$ test (threshold $T>11 ; P<0.001$, two tail) and (ii) tested for significant differences between MOR knockout and control groups using $t$ test (threshold $T>2$; significance/alpha level 0.05 , one tail). All results were familywise error rate (EWER) corrected following clustering with significance/alpha level $0.05 .^{53}$ For $3 \mathrm{D}$ representation of the mouse brain for all the data, we used Mango (http://ric. uthscsa.edu/mango/) and BrainNet Viewer (https://www. nitrc.org/projects/bnv/) tools.

\section{ASSOCIATED CONTENT}

\section{S Supporting Information}

The Supporting Information is available free of charge on the ACS Publications website at DOI: 10.1021/acsptsci.9b00021.

Number of FC alterations induced by oxycodone for each component in CTL animals; quantification of oxycodone-induced reduction of FC between voxels from the PAG seed and voxels from 8 other seeds; quantification of oxycodone-induced reduction of FC between voxels from the NAc seed and voxels from 22 other seeds; additional figures (PDF)

\section{AUTHOR INFORMATION}

\section{Corresponding Authors}

*Tel.: 514 761-6131 ext: 3175. Fax: 514 762-3033. E-mail: brigitte.kieffer@douglas.mcgill.ca.

*Tel.: 514 761-6131 ext: 4772. E-mail: emmanuel.darcq@ douglas.mcgill.ca.

\section{ORCID}

Emmanuel Darcq: 0000-0002-8377-8015

\section{Author Contributions}

Md.T.N., B.L.K., and E.D. designed the study; D.W.P. generated and genotyped the mice, Md.T.N., J.P.S., A.T.E., M.M., W.M., and E.D. acquired the data, Md.T.N., J.P.S., and E.D. performed the analysis; P.K. helped and assisted the BOLD analysis. Md.T.N., B.L.K., and E.D. interpreted data and wrote the manuscript.

\section{Notes}

The authors declare no competing financial interest.

\section{ACKNOWLEDGMENTS}

We thank the staff at the animal facility of the Neurophenotyping Center and of the Brain Imaging Center at the Douglas Research Center (Montréal, Canada) and Axel Mathieu for his assistance with MRI acquisition. This work was supported by National Institute of Health (National Institute of Drug Abuse Grant No. 05010 to B.L.K. and National Institute on Alcohol Abuse and Alcoholism, Grant No. 16658 to B.L.K.), the Canada Fund for Innovation and the Canada Research Chairs to B.L.K. We also thank Alkermes for their support. The NIDA Supply Drug Program generously provided oxycodone.

\section{LIST OF ABBREVIATIONS}

BLA, Basolateral amygdalar nucleus; BMA, Basomedial amygdalar nucleus; CP, Caudoputamen; CEA, Central amygdalar nucleus; COA, Cortical amygdalar area; ENT, Entorhinal area; Hipp, Hippocampus; IPN, Interpeduncular nucleus; LHA, Lateral hypothalamic area; MRN, Midbrain reticular nucleus; MY, Medulla; PG, Pontine gray; PIR, Piriform area; POR, Superior olivary complex; PRN, Pontine reticular nucleus/caudal part; SC, Superior colliculus; SI, Substantia innominata; SNr, Substantia nigra; TRN, Tegmental reticular nucleus; vmHy, Ventromedial hypothalamic nucleus; vmTh, Ventral medial nucleus of the thalamus; Th, Thalamus

\section{REFERENCES}

(1) Dice, T. J., and Mead, T. (2018) Oxycodone. In Stat Pearls, StatPearls Publishing StatPearls Publishing LLC., Treasure Island (FL).

(2) Gkegkes, I. D., Minis, E. E., and Iavazzo, C. (2018) Oxycodone/ naloxone in postoperative pain management of surgical patients. $J$. Opioid Manag 14 (1), 52-60.

(3) Pergolizzi, J. V., Jr., Taylor, R., Jr., LeQuang, J. A., and Raffa, R. B. (2018) Managing severe pain and abuse potential: the potential impact of a new abuse-deterrent formulation oxycodone/naltrexone extended-release product. J. Pain Res. 11, 301-311. 
(4) Schmidt-Hansen, M., Bennett, M. I., Arnold, S., Bromham, N., and Hilgart, J. S. (2018) Efficacy, tolerability and acceptability of oxycodone for cancer-related pain in adults: an updated Cochrane systematic review. BMJ. Support Palliat Care 8 (2), 117-128.

(5) Cheung, C. W., Ching Wong, S. S., Qiu, Q., and Wang, X. (2017) Oral Oxycodone for Acute Postoperative Pain: A Review of Clinical Trials. Pain Physician 20 (2S), SE33-SE52.

(6) Kalso, E. (2005) Oxycodone. J. Pain Symptom Manage. 29 (5), S47-56.

(7) Olkkola, K. T., Kontinen, V. K., Saari, T. I., and Kalso, E. A. (2013) Does the pharmacology of oxycodone justify its increasing use as an analgesic? Trends Pharmacol. Sci. 34 (4), 206-14.

(8) Poyhia, R., Vainio, A., and Kalso, E. (1993) A review of oxycodone's clinical pharmacokinetics and pharmacodynamics. J. Pain Symptom Manage. 8 (2), 63-7.

(9) Stoops, W. W., Hatton, K. W., Lofwall, M. R., Nuzzo, P. A., and Walsh, S. L. (2010) Intravenous oxycodone, hydrocodone, and morphine in recreational opioid users: abuse potential and relative potencies. Psychopharmacology 212 (2), 193-203.

(10) Comer, S. D., Metz, V. E., Cooper, Z. D., Kowalczyk, W. J., Jones, J. D., Sullivan, M. A., Manubay, J. M., Vosburg, S. K., Smith, M. E., Peyser, D., and Saccone, P. A. (2013) Comparison of a drug versus money and drug versus drug self-administration choice procedure with oxycodone and morphine in opioid addicts. Behav. Pharmacol. 24 (5-6), 504-16.

(11) Kolodny, A., Courtwright, D. T., Hwang, C. S., Kreiner, P., Eadie, J. L., Clark, T. W., and Alexander, G. C. (2015) The prescription opioid and heroin crisis: a public health approach to an epidemic of addiction. Annu. Rev. Public Health 36, 559-74.

(12) World Health Organization (2017) Curbing prescription opioid dependency. Bull. World Health Organ 95 (5), 318-319.

(13) Darcq, E., and Kieffer, B. L. (2018) Opioid receptors: drivers to addiction? Nat. Rev. Neurosci. 19 (8), 499-514.

(14) Siuda, E. R., Carr, R., 3rd, Rominger, D. H., and Violin, J. D. (2017) Biased mu-opioid receptor ligands: a promising new generation of pain therapeutics. Curr. Opin. Pharmacol. 32, 77-84.

(15) Moningka, H., Lichenstein, S., Worhunsky, P. D., DeVito, E. E., Scheinost, D., and Yip, S. W. (2019) Can neuroimaging help combat the opioid epidemic? A systematic review of clinical and pharmacological challenge fMRI studies with recommendations for future research. Neuropsychopharmacology 44 (2), 259-273.

(16) Matthes, H. W., Maldonado, R., Simonin, F., Valverde, O., Slowe, S., Kitchen, I., Befort, K., Dierich, A., Le Meur, M., Dolle, P., Tzavara, E., Hanoune, J., Roques, B. P., and Kieffer, B. L. (1996) Loss of morphine-induced analgesia, reward effect and withdrawal symptoms in mice lacking the mu-opioid-receptor gene. Nature 383 (6603), 819-23.

(17) Huang, W., Manglik, A., Venkatakrishnan, A. J., Laeremans, T., Feinberg, E. N., Sanborn, A. L., Kato, H. E., Livingston, K. E., Thorsen, T. S., Kling, R. C., Granier, S., Gmeiner, P., Husbands, S. M., Traynor, J. R., Weis, W. I., Steyaert, J., Dror, R. O., and Kobilka, B. K. (2015) Structural insights into micro-opioid receptor activation. Nature 524 (7565), 315-21.

(18) Williams, J. T., Ingram, S. L., Henderson, G., Chavkin, C., von Zastrow, M., Schulz, S., Koch, T., Evans, C. J., and Christie, M. J. (2013) Regulation of mu-opioid receptors: desensitization, phosphorylation, internalization, and tolerance. Pharmacol. Rev. 65 (1), 22354.

(19) Erbs, E., Faget, L., Scherrer, G., Matifas, A., Filliol, D., Vonesch, J. L., Koch, M., Kessler, P., Hentsch, D., Birling, M. C., Koutsourakis, M., Vasseur, L., Veinante, P., Kieffer, B. L., and Massotte, D. (2015) A mu-delta opioid receptor brain atlas reveals neuronal co-occurrence in subcortical networks. Brain Struct. Funct. 220 (2), 677-702.

(20) Le Merrer, J., Becker, J. A., Befort, K., and Kieffer, B. L. (2009) Reward processing by the opioid system in the brain. Physiol. Rev. 89 (4), 1379-412.

(21) Borsook, D., Hargreaves, R, and Becerra, L. (2011) Can. Functional Magnetic Resonance Imaging Improve Success Rates in CNS Drug Discovery? Expert Opin. Drug Discovery 6 (6), 597-617.
(22) Ferrari, L., Turrini, G., Crestan, V., Bertani, S., Cristofori, P., Bifone, A., and Gozzi, A. (2012) A robust experimental protocol for pharmacological fMRI in rats and mice. J. Neurosci. Methods 204 (1), $9-18$.

(23) Jonckers, E., Shah, D., Hamaide, J., Verhoye, M., and Van der Linden, A. (2015) The power of using functional fMRI on small rodents to study brain pharmacology and disease. Front. Pharmacol. 6, 231.

(24) Jonckers, E., Van der Linden, A., and Verhoye, M. (2013) Functional magnetic resonance imaging in rodents: an unique tool to study in vivo pharmacologic neuromodulation. Curr. Opin. Pharmacol. 13 (5), 813-20.

(25) Iriah, S. C., Trivedi, M., Kenkel, W., Grant, S. E., Moore, K., Yee, J. R., Madularu, D., Kulkarni, P., and Ferris, C. F. (2019) Oxycodone Exposure: A Magnetic Resonance Imaging Study in Response to Acute and Chronic Oxycodone Treatment in Rats. Neuroscience 398, 88-101.

(26) Moore, K., Madularu, D., Iriah, S., Yee, J. R., Kulkarni, P., Darcq, E., Kieffer, B. L., and Ferris, C. F. (2016) BOLD Imaging in Awake Wild-Type and Mu-Opioid Receptor Knock-Out Mice Reveals On-Target Activation Maps in Response to Oxycodone. Front. Neurosci. 10, 471.

(27) Reichling, D. B., Kwiat, G. C., and Basbaum, A. I. (1988) Anatomy, physiology and pharmacology of the periaqueductal gray contribution to antinociceptive controls. Prog. Brain Res. 77, 31-46.

(28) Basbaum, A. I., Bautista, D. M., Scherrer, G., and Julius, D. (2009) Cellular and molecular mechanisms of pain. Cell 139 (2), 267-84.

(29) Hu, H. (2016) Reward and Aversion. Annu. Rev. Neurosci. 39, 297-324.

(30) Koob, G. F., and Volkow, N. D. (2016) Neurobiology of addiction: a neurocircuitry analysis. Lancet Psychiatry 3 (8), 760-73.

(31) Fields, H. L., and Margolis, E. B. (2015) Understanding opioid reward. Trends Neurosci. 38 (4), 217-25.

(32) Badiani, A., Belin, D., Epstein, D., Calu, D., and Shaham, Y. (2011) Opiate versus psychostimulant addiction: the differences do matter. Nat. Rev. Neurosci. 12 (11), 685-700.

(33) Ehrlich, A. T., Maroteaux, G., Robe, A., Venteo, L., Nasseef, M. T., van Kempen, L. C., Mechawar, N., Turecki, G., Darcq, E., and Kieffer, B. L. (2018) Expression map of 78 brain-expressed mouse orphan GPCRs provides a translational resource for neuropsychiatric research. Communications Biology 1 (1), 102.

(34) Boulos, L. J., Nasseef, M. T., McNicholas, M., Mechling, A., Harsan, L. A., Darcq, E., Ben Hamida, S., and Kieffer, B. L. (2019) TouchScreen-based phenotyping: altered stimulus/reward association and lower perseveration to gain a reward in mu opioid receptor knockout mice. Sci. Rep. 9 (1), 4044.

(35) Becerra, L., Upadhyay, J., Chang, P. C., Bishop, J., Anderson, J., Baumgartner, R., Schwarz, A. J., Coimbra, A., Wallin, D., Nutile, L., George, E., Maier, G., Sunkaraneni, S., Iyengar, S., Evelhoch, J. L., Bleakman, D., Hargreaves, R., and Borsook, D. (2013) Parallel buprenorphine phMRI responses in conscious rodents and healthy human subjects. J. Pharmacol. Exp. Ther. 345 (1), 41-51.

(36) Liu, C. H., Greve, D. N., Dai, G., Marota, J. J., and Mandeville, J. B. (2007) Remifentanil administration reveals biphasic phMRI temporal responses in rat consistent with dynamic receptor regulation. NeuroImage 34 (3), 1042-53.

(37) Shah, Y. B., Haynes, L., Prior, M. J., Marsden, C. A., Morris, P. G., and Chapman, V. (2005) Functional magnetic resonance imaging studies of opioid receptor-mediated modulation of noxious-evoked BOLD contrast in rats. Psychopharmacology 180 (4), 761-73.

(38) Bossert, J. M., Adhikary, S., St. Laurent, R., Marchant, N. J., Wang, H. L., Morales, M., and Shaham, Y. (2016) Role of projections from ventral subiculum to nucleus accumbens shell in contextinduced reinstatement of heroin seeking in rats. Psychopharmacology 233 (10), 1991-2004.

(39) Hoehner, P. J., Whitson, J. T., Kirsch, J. R., and Traystman, R. J. (1993) Effect of intracarotid and intraventricular morphine on 
regional cerebral blood flow and metabolism in pentobarbitalanesthetized dogs. Anesth. Analg. 76 (2), 266-73.

(40) Shih, Y. Y., Chiang, Y. C., Shyu, B. C., Jaw, F. S., Duong, T. Q., and Chang, C. (2012) Endogenous opioid-dopamine neurotransmission underlie negative CBV fMRI signals. Exp. Neurol. 234 (2), 3828.

(41) Gorka, S. M., Fitzgerald, D. A., de Wit, H., Angstadt, M., and Phan, K. L. (2014) Opioid modulation of resting-state anterior cingulate cortex functional connectivity. J. Psychopharmacol. (London, U. K.) 28 (12), 1115-24.

(42) Navratilova, E., Atcherley, C. W., and Porreca, F. (2015) Brain Circuits Encoding Reward from Pain Relief. Trends Neurosci. 38 (11), 741-750.

(43) Ehrlich, A. T., Kieffer, B. L., and Darcq, E. (2019) Current strategies toward safer mu opioid receptor drugs for pain management. Expert Opin. Ther. Targets 23, 315.

(44) Darcq, E., Befort, K., Koebel, P., Pannetier, S., Mahoney, M. K., Gaveriaux-Ruff, C., Hanauer, A., and Kieffer, B. L. (2012) RSK2 signaling in medial habenula contributes to acute morphine analgesia. Neuropsychopharmacology 37 (5), 1288-96.

(45) Charbogne, P., Gardon, O., Martin-Garcia, E., Keyworth, H. L., Matsui, A., Mechling, A. E., Bienert, T., Nasseef, T., Robe, A., Moquin, L., Darcq, E., Ben Hamida, S., Robledo, P., Matifas, A., Befort, K., Gaveriaux-Ruff, C., Harsan, L. A., von Elverfeldt, D., Hennig, J., Gratton, A., Kitchen, I., Bailey, A., Alvarez, V. A., Maldonado, R., and Kieffer, B. L. (2017) Mu Opioid Receptors in Gamma-Aminobutyric Acidergic Forebrain Neurons Moderate Motivation for Heroin and Palatable Food. Biol. Psychiatry 81 (9), 778-788.

(46) Mechling, A. E., Hubner, N. S., Lee, H. L., Hennig, J., von Elverfeldt, D., and Harsan, L. A. (2014) Fine-grained mapping of mouse brain functional connectivity with resting-state fMRI. NeuroImage 96, 203-15.

(47) Dumais, K. M., Kulkarni, P. P., Ferris, C. F., and Veenema, A. H. (2017) Sex differences in neural activation following different routes of oxytocin administration in awake adult rats. Psychoneuroendocrinology $81,52-62$.

(48) Chakravarty, M. M., Steadman, P., van Eede, M. C., Calcott, R. D., Gu, V., Shaw, P., Raznahan, A., Collins, D. L., and Lerch, J. P. (2013) Performing label-fusion-based segmentation using multiple automatically generated templates. Human brain mapping 34 (10), 2635-54.

(49) Himberg, J., Hyvarinen, A., and Esposito, F. (2004) Validating the independent components of neuroimaging time series via clustering and visualization. NeuroImage 22 (3), 1214-22.

(50) Arefin, T. M., Mechling, A. E., Meirsman, A. C., Bienert, T., Hubner, N. S., Lee, H. L., Ben Hamida, S., Ehrlich, A., Roquet, D., Hennig, J., von Elverfeldt, D., Kieffer, B. L., and Harsan, L. A. (2017) Remodeling of Sensorimotor Brain Connectivity in Gpr88-Deficient Mice. Brain connectivity 7 (8), 526-540.

(51) Ben Hamida, S., Mendonca-Netto, S., Arefin, T. M., Nasseef, M. T., Boulos, L. J., McNicholas, M., Ehrlich, A. T., Clarke, E., Moquin, L., Gratton, A., Darcq, E., Harsan, L. A., Maldonado, R., and Kieffer, B. L. (2018) Increased Alcohol Seeking in Mice Lacking Gpr88 Involves Dysfunctional Mesocorticolimbic Networks. Biol. Psychiatry 84 (3), 202-212.

(52) Takasu, K., Ogawa, K., Nakamura, A., Kanbara, T., Ono, H., Tomii, T., Morioka, Y., Hasegawa, M., Shibasaki, M., Mori, T., Suzuki, T., and Sakaguchi, G. (2015) Enhanced GABAergic synaptic transmission at VLPAG neurons and potent modulation by oxycodone in a bone cancer pain model. British journal of pharmacology 172 (8), 2148-64.

(53) Nichols, T., and Hayasaka, S. (2003) Controlling the familywise error rate in functional neuroimaging: a comparative review. Statistical methods in medical research 12 (5), 419-46. 